\title{
Demonstrating the capacity of the National Advisory Committee on Immunization for timely responses to post-market vaccine monitoring signals: Canada's experience with the live- attenuated influenza vaccine
}

\author{
Linlu Zhao ${ }^{1 *}$, Kelsey Young ${ }^{1}$, Althea House ${ }^{1}$, Rob Stirling ${ }^{1}$, Matthew Tunis ${ }^{1}$
}

\begin{abstract}
Over the last several years, the recommended use of the live-attenuated influenza vaccine (LAIV) for children has evolved in the United States (US) in response to evidence of a potential decrease in LAIV effectiveness based on post-market monitoring. These issues were not observed in Canada or elsewhere; consequently, recommendations from Canada's National Advisory Committee on Immunization (NACl) and the US Advisory Committee on Immunization Practices (ACIP) on whether to use LAIV differed for two influenza seasons (2016-2017 and 2017-2018).

This retrospective describes how $\mathrm{NACl}$ arrived at its recommendations in response to post-market signals of reduced LAIV performance from the US in 2013-2014 and again in 2015-2016. NACl's experience with LAIV marks the first time in Canada where a preferential recommendation on the use of an influenza vaccine in a routine immunization program was reversed. This experience highlights the importance of ongoing post-market monitoring of vaccines, international collaboration and careful consideration of local context to inform vaccine recommendations. NACl's capacity for timely responses to post-market vaccine performance signals will facilitate responsiveness to similar post-market monitoring signals from the coronavirus disease 2019 (COVID-19) vaccines.
\end{abstract}

This work is licensed under a Creative Commons Attribution 4.0 International License.

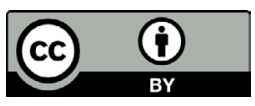

Affiliation

${ }^{1}$ Public Health Agency of Canada

*Correspondence:

linlu.zhao@canada.ca

Suggested citation: Zhao L, Young K, House A, Stirling R, Tunis M. Demonstrating the capacity of the National Advisory Committee on Immunization for timely responses to post-market vaccine monitoring signals: Canada's experience with the live-attenuated influenza vaccine. Can Commun Dis Rep 2021;47(5/6):279-84.

https://doi.org/10.14745/ccdr.v47i56a06

Keywords: influenza, LAIV, nasal spray, post-market surveillance, vaccine effectiveness

\section{Introduction}

The World Health Organization recommends that every country have a National Immunization Technical Advisory Group (NITAG) of experts to provide independent, evidence-informed vaccine recommendations $(1,2)$. Canada's National Advisory Committee on Immunization (NACl), an external advisory body to the Public Health Agency of Canada (PHAC), is one of the longest standing NITAGs in the world. NACl has been providing ongoing and timely expert and evidence-based advice on the use of vaccines to protect Canadians for over 50 years.
$\mathrm{NACl}$ 's recommendations are developed using an evidence-based process, which broadly involves the sequential stages of evidence gathering, synthesis and translation to recommendations (3). Triggers for $\mathrm{NACl}$ guidance development include-but are not limited to-authorization of new vaccines in the Canadian market; changes to indications for vaccine use; detection of vaccine safety or performance signals through post-market monitoring; the publication of pivotal new research; specific concerns raised by provincial and territorial immunization programs; and significant changes in international guidance. 
Findings of suboptimal effectiveness of live-attenuated influenza vaccine (LAIV) in the United States (US) in 2014 and again in 2016 were triggers for $\mathrm{NACl}$ to review and deliberate on their guidance on the use of the LAIV in Canada.

This article documents NACl's responses to these signals of potentially decreased LAIV effectiveness in the US and highlights the importance of ongoing post-market monitoring of vaccines.

\section{Triggers and responses}

LAIV has been demonstrated to be safe and efficacious through clinical trials, but real-world evidence collected after LAIV was made available for use showed that it may be less effective in some contexts than the more established inactivated influenza vaccines (IIVs).

LAIV was first marketed in Canada as a trivalent formulation for individuals aged 2-59 years in June 2010, seven years after the vaccine was first marketed in the US. It was offered in various provinces and territories as part of publicly funded immunization programs beginning in 2012-2013. The trivalent formulation of LAIV was replaced with the quadrivalent formulation in Canada starting in the 2014-2015 influenza season.

In 2011, NACI made a preferential recommendation for the use of trivalent LAIV over trivalent IIV for children and adolescents aged 2-17 years for the 2011-2012 season (quadrivalent influenza vaccines were not available in Canada at the time) (4).
This decision was based upon favourable efficacy data from clinical trials and post-market safety data, with acknowledgement of stronger evidence for superior efficacy in younger children (younger than six years old) than for older children. Based on the efficacy data, in June 2014 the Advisory Committee on Immunization Practices (ACIP) - the US counterpart to $\mathrm{NACl}$-recommended the preferential use of quadrivalent LAIV over trivalent or quadrivalent IIV for the 2014-2015 season for children 2-8 years old. The upper age limit of ACIP's recommendation was chosen based on programmatic consistency (i.e. in the US, eight years of age is also the upper age limit for the receipt of two doses of influenza vaccine in a previously unvaccinated child) (5). These preferential LAIV use recommendations by $\mathrm{NACl}$ and ACIP were in place when findings of reduced LAIV effectiveness against influenza $A(H 1 N 1)$ in the 2013-2014 season in the US came to light $(4,5)$.

Figure 1 provides a visual summary of the milestones in the development of recommendations on the use of LAIV in Canada and the US. Table 1 provides a verbal summary of these events.

\section{First trigger for potential NACI review of LAIV guidance}

In October 2014, a few months after ACIP made its preferential recommendation, post-market monitoring studies found evidence of low to no LAIV effectiveness against influenza $\mathrm{A}(\mathrm{H} 1 \mathrm{~N} 1)$ in US children and adolescents 2-17 years old in the 2013-2014 season; this evidence was presented to ACIP (6).

Figure 1: Milestones of recommendations on LAIV use in Canada and the US

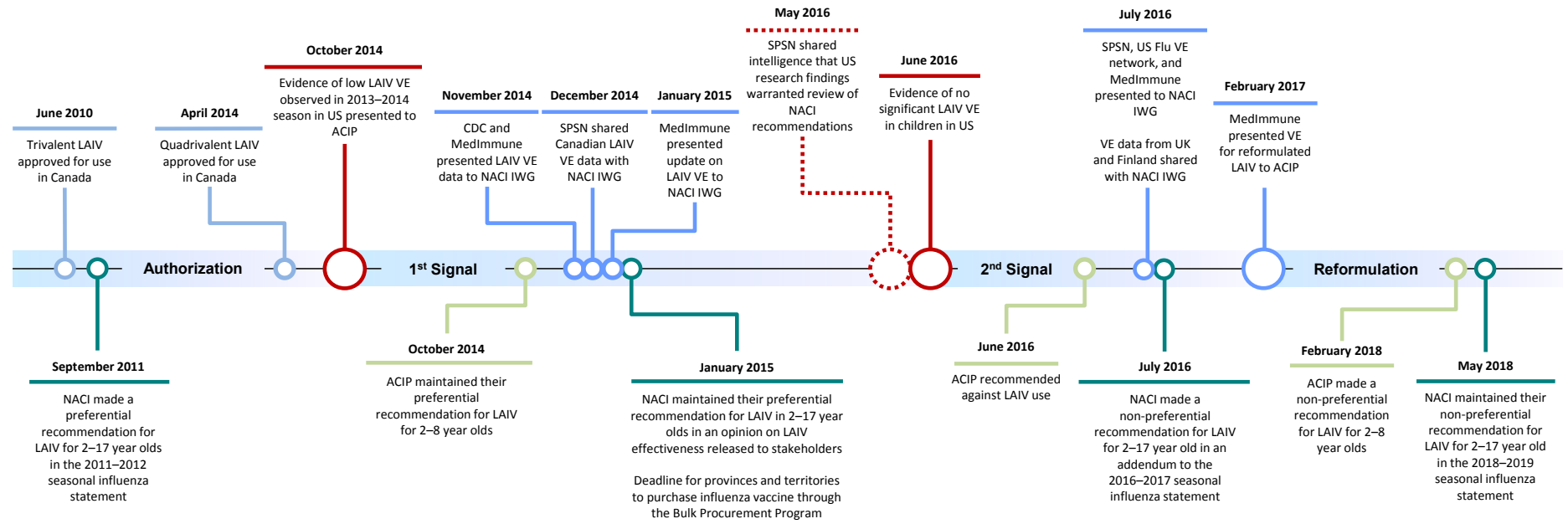

Abbreviations: ACIP, Advisory Committee on Immunization Practices; CDC, United States Centers for Disease Control and Prevention; IWG, Influenza Working Group AIV, live-attenuated influenza vaccine; NACl, National Advisory Committee on Immunization; SPSN, Sentinel Practitioner Surveillance Network; UK, United Kingdom; US, United States; $V E$, vaccine effectiveness 
Table 1: Timeline of $\mathrm{NACl}$ responses to the LAIV performance signals from the US

\begin{tabular}{|c|c|}
\hline Date & Response \\
\hline First signal & $\begin{array}{l}\text { On October 29, 2014, evidence of low LAIV } \\
\text { effectiveness for the 2013-2014 influenza season } \\
\text { in the US was presented to ACIP. }\end{array}$ \\
\hline $\begin{array}{l}\text { November 18, } \\
2014\end{array}$ & $\begin{array}{l}\text { LAIV effectiveness data from the US for the 2013- } \\
2014 \text { season were presented to a joint meeting of } \\
\text { NACI, NACI IWG and CIC (representing provincial } \\
\text { and territorial immunization programs). }\end{array}$ \\
\hline $\begin{array}{l}\text { Late } \\
\text { November } \\
\text { to December } \\
2014\end{array}$ & $\begin{array}{l}\text { Canada's SPSN shared LAIV effectiveness data for the } \\
\text { 2013-2014 season with NACI. Based on the available } \\
\text { evidence, NACI concluded that no change will be } \\
\text { made to the recommendation on LAIV use for the } \\
\text { 2015-2016 season. }\end{array}$ \\
\hline $\begin{array}{l}\text { January 26, } \\
2015\end{array}$ & $\begin{array}{l}\text { NACI's position on LAIV use in response to the first } \\
\text { signal was distributed to provincial and territorial } \\
\text { stakeholders. }\end{array}$ \\
\hline Second signal & $\begin{array}{l}\text { On June 22, 2016, ACIP recommended against } \\
\text { LAIV use for the 2016-2017 season based on } \\
\text { evidence of low LAIV effectiveness for two } \\
\text { influenza A(H1N1)pdm09 predominate seasons } \\
\text { (2013-2014 and 2015-2016) in the US. }\end{array}$ \\
\hline July 4, 2016 & $\begin{array}{l}\text { PHAC hosted a teleconference with } \mathrm{CIC} \text { to discuss } \\
\text { the LAIV effectiveness signal from the US and NACI's } \\
\text { plans to review the available evidence. NACI's } \\
\text { secretariat at PHAC started synthesizing available } \\
\text { post-2009 LAIV effectiveness data. }\end{array}$ \\
\hline July 19, 2016 & $\begin{array}{l}\text { Post-2009 LAIV effectiveness data, including data } \\
\text { from Canada, the US and other jurisdictions for the } \\
2015-2016 \text { influenza season, were presented to } \\
\text { NACI's IWG for deliberation. Based on the available } \\
\text { evidence, the IWG proposed a recommendation } \\
\text { change for NACl's consideration. }\end{array}$ \\
\hline July 26, 2016 & $\begin{array}{l}\mathrm{NACl} \text { concluded that the available evidence did not } \\
\text { support a recommendation for the preferential use of } \\
\text { LAIV over IIV, but LAIV remained an influenza vaccine } \\
\text { option for children. }\end{array}$ \\
\hline July 29, 2016 & $\begin{array}{l}\text { NACI's position on LAIV use in response to the } \\
\text { second signal was distributed to provincial and } \\
\text { territorial stakeholders. }\end{array}$ \\
\hline
\end{tabular}

Abbreviations: ACIP, Advisory Committee on Immunization Practices; $\mathrm{ClC}$, Canadian Immunization Committee; IIV, inactivated influenza vaccine; IWG, Influenza Working Group; LAIV, live-attenuated influenza vaccine; $\mathrm{NACl}$, National Advisory Committee on Immunization; PHAC, Public Health Agency of Canada; SPSN, Sentinel Practitioner Surveillance Network; US, United States

The low LAIV performance observed in the US led NACI to initiate a rapid review of the evidence to determine whether there was a need to revise its recommendation on how LAIV should continue to be used in Canada. The evidence review needed to be rapid to inform provincial and territorial procurement decisions that would be made early in 2015 for the 2015-2016 influenza season. By November 2014, invited speakers from the US Centers for Disease Control and Prevention (CDC) and Medlmmune, the manufacturer of LAIV, had presented their US LAIV effectiveness data to $\mathrm{NACl}$ and its Influenza Working Group (IWG). At the time, Medlmmune proposed that the reduced effectiveness against influenza $A(H 1 N 1)$ seen in the US may have been due to the vulnerability to heat degradation of the A/California/7/2009(H1N1)pdm09-like strain present in the vaccine, which may have occurred during vaccine distribution. In December 2014, Canada's Sentinel Practitioner Surveillance Network (SPSN) shared with NACl unpublished Canadian LAIV effectiveness data from the 2013-2014 season that showed that the effectiveness of LAIV was similar to IIV, which differed from the US data.

After reviewing this effectiveness data from the 2013-2014 season, $\mathrm{NACl}$ published an opinion statement in late January 2015 , in time to inform provincial and territorial procurement decisions for the upcoming 2015-2016 season. The opinion statement indicated that no change would be made to NACl's preferential recommendation on LAIV use (7). NACl's decision also took into consideration factors other than vaccine effectiveness, including the different LAIV formulations used in the US (quadrivalent) and Canada (trivalent) in 2013-2014; differences in the temperature-controlled vaccine distribution between the two countries; and the fact that the low LAIV effectiveness seen in the US studies for the 2013-2014 season was not seen in Canadian post-market LAIV effectiveness data from SPSN for the same season (8). In contrast, in February 2015, ACIP reversed its preferential LAIV use recommendation to indicate either LAIV or IIV were appropriate vaccine options in children aged 2-8 years for the 2015-2016 season (9).

As a result of the manufacturer's thermal stability investigations, the $A(\mathrm{H} 1 \mathrm{~N} 1)$ pdm09 component of LAIV was changed for the 2014-2015 season from the A/California/7/2009 strain to the antigenically similar and more heat stable A/Bolivia/559/2013 strain. Whether the strain change for LAIV improved its performance for the 2014-2015 influenza season as compared to the inactivated vaccine was inconclusive as the season was dominated by antigenically drifted $\mathrm{A}(\mathrm{H} 3 \mathrm{~N} 2)$ viruses. Both LAIV and IIV performed poorly for the 2014-2015 season; a majority of studies found no evidence that either type of vaccine protected against any influenza and influenza $A(H 3 N 2)(10)$.

\section{Second trigger for potential NACI review of guidance}

Post-market monitoring studies completed in the US at the end of the 2015-2016 season again found low vaccine effectiveness for LAIV, but not IIV, against influenza A(H1N1) in children and adolescents aged 2-17 years (11). On June 22, 2016, ACIP recommended against the use of LAIV for the 2016-2017 season. ACIP's decision was driven by the reduced effectiveness observed for LAIV against A(H1N1) in the US over the 2013-2014 and 2015-2016 seasons when predominately influenza $A(H 1 N 1)$ pdm09-like viruses circulated $(12,13)$. This decision garnered widespread attention and generated a high degree of interest from Canadian stakeholders. 
The new US LAIV data and ACIP's recommendation against the use of LAIV in the US raised concerns regarding the use of LAIV in Canada from provincial and territorial immunization programs, which had already procured LAIV. All of these factors were triggers for $\mathrm{NACl}$ to once again review its guidance on LAIV use in Canada.

NACl's challenge in response to this trigger was the time constraint to provide a very rapid evidence-informed decision on the use of the already procured LAIV mere months from the start of provincial and territorial immunization campaigns (typically October) for the upcoming season. On July 4, 2016, PHAC held a meeting with stakeholders from provincial and territorial immunization programs to brief them about the new LAIV effectiveness data from the US and to inform them of NACl's planned activities to review additional Canadian and international effectiveness data for LAIV to inform NACl's review of its LAIV recommendations.

On July 19, 2016, the NACI IWG reviewed unpublished postmarket monitoring data on LAIV effectiveness for the 2015-2016 influenza season from six sources: Canada's SPSN; the US Influenza Vaccine Effectiveness Network (US Flu VE Network); the US Department of Defense; the United Kingdom's influenza vaccine effectiveness network; the National Institute for Health and Welfare of Finland; and Medlmmune. The IWG also reviewed published and unpublished post-2009 pandemic data to assess the trend of LAIV effectiveness over the influenza seasons since the $A(H 1 N 1) p d m 09$ pandemic strain displaced the pre-pandemic influenza $A(H 1 N 1)$ strains. These included findings from two Canadian cluster randomized controlled trials that did not find reduced LAIV effectiveness for the 2013-2014 season in Canada $(14,15)$, which aligned with findings from SPSN for that season.

Following reviews of the available evidence, which showed LAIV providing protection against influenza comparable to that afforded by IIV, and discussions with various jurisdictions, the IWG recommended that $\mathrm{NACl}$ change its recommendations on the use of LAIV. On July 26, 2016, based on the advice from the IWG and after considering all the available evidence, $\mathrm{NACl}$ concluded that the available evidence no longer supported a recommendation for the preferential use of LAIV over IIV, but that LAIV remained an option for the annual influenza vaccination of children aged 2-17 years (6). Although the US data showing LAIV effectiveness to be comparable or lower than IIV effectiveness contributed to NACl's revised, non-preferential recommendation on LAIV use, the reduced effectiveness seen in the US for the 2015-2016 season was again not observed in Canada or other countries that investigated the issue (10). The difference in LAIV performance data from Canada and other international jurisdictions compared to data from the US played an important role in informing NACl's decision to continue to recommend LAIV use in Canada for the 2016-2017 season (16). NACl's official position was communicated to stakeholders in late July $2016(6,10,16)$. The effectiveness data supporting NACl's decision has been detailed elsewhere (10).

\section{Epilogue}

The poor performance of the $A(\mathrm{H} 1 \mathrm{~N} 1)$ component of LAIV in the 2015-2016 influenza season was attributed to reduced replicative fitness of the vaccine $A(\mathrm{H} 1 \mathrm{~N} 1)$ pdm09-like strain. Similar to the 2013-2014 season, differences in the temperature-controlled vaccine distribution between Canada and the US could be a factor in the differential performance of LAIV in the two countries for the 2015-2016 season. Due to the finding of reduced replicative fitness, the $A(\mathrm{H} 1 \mathrm{~N} 1) \mathrm{pdm} 09-$ like strain in the 2015-2016 vaccine formulation (A/Bolivia/559/2013) was replaced with a new strain (A/Slovenia/2903/2015) for the 2017-2018 season (13).

ACIP maintained their recommendation against LAIV use for the 2017-2018 season. However, in February 2018, ACIP voted to reinstate LAIV as a vaccine option for the 2018-2019 season, with no preference given to LAIV or IIV for the paediatric age group, based on data provided from the manufacturer suggesting that the new $\mathrm{A}(\mathrm{H} 1 \mathrm{~N} 1)$ pdm09-like strain (A/Slovenia/2903/2015) has improved replicative fitness over previous $A(\mathrm{H} 1 \mathrm{~N} 1)$ pdm09-like vaccine strains in LAIV (12). This non-preferential recommendation is maintained by ACIP in their latest statement for the 2020-2021 season (17). NACl's recommendation for the non-preferential use of LAIV in children and adolescents aged 2-17 years remains unchanged going into the 2021-2022 influenza season (18).

\section{Discussion}

$\mathrm{NACl}$ and $\mathrm{ACIP}$ arrived at different conclusions on the use of LAIV in response to the post-market LAIV performance signals from the US for the 2013-2014 and 2015-2016 seasons, but differing recommendations by NITAGs are not unusual. NITAGs consider a multitude of factors when making their recommendations, including vaccine characteristics (efficacy, effectiveness, immunogenicity and safety), local burden of disease, and vaccine effectiveness data. Based on this complex multifactorial analysis and differential weighting of these factors in their analyses, it is not surprising that NITAGs often arrive at different conclusions about which immunization strategy will best address the needs of their specific country.

NACl's rapid evidence appraisal and decision-making in response to the low LAIV effectiveness data from the US was facilitated by several critical factors. Being able to depend on seasonal influenza vaccine effectiveness assessments from established Canadian influenza surveillance networks was one facilitator. Canada's SPSN provided a near real-time assessment of their data to $\mathrm{NACl}$ and PHAC to inform NACl's deliberations on LAIV use in children.

Another facilitator was having Canadian influenza vaccine effectiveness data from studies that were specifically designed and appropriately powered to compare LAIV and IIV, such as 
the cluster randomized clinical trials that corroborated the SPSN surveillance findings from the 2013-2014 season. (SPSN was not designed to compare the effectiveness of specific vaccine products.)

A third facilitator was collaboration with international and industry partners. Sharing of intelligence on emerging signals and unpublished data was pivotal to informing NACl's guidance development with the best available evidence in these timesensitive situations. There were also several critical factors that contributed to successfully meeting the evidence-toguidance challenge from an operational perspective. These included having established processes to facilitate stakeholder engagement and mobilizing surge capacity within PHAC to provide technical and logistical support to $\mathrm{NACl}$.

\section{Conclusion}

NACl's response to the post-market LAIV performance signals from the US represents an example of its capacity to respond rapidly and comprehensively to the dynamic landscape of international vaccine research and infectious disease epidemiology. These responses highlight the importance of establishing and leveraging existing channels for intelligence sharing and knowledge exchange with evidence producers, partners and users and the importance of considering evidence from multiple sources and the local context for informing decision-making. NACl's experience with and capacity for timely responses to post-market vaccine performance signals will facilitate responsiveness to similar post-market monitoring signals from the coronavirus disease 2019 (COVID-19) vaccines.

\section{Authors' statement}

LZ - Conceptualization, writing-original draft, writing-review and editing

$\mathrm{KY}$ - Conceptualization, writing-review and editing

$\mathrm{AH}$ - Conceptualization, writing-review and editing

RS - Conceptualization, writing-review and editing

MT - Conceptualization, writing-review and editing

\section{Competing interests}

None.

\section{Funding}

This work was supported by the Public Health Agency of Canada.

\section{References}

1. Adjagba A, MacDonald NE, Ortega-Pérez I, Duclos P; 2016 Global NITAG Network Meeting Participants. Strengthening and sustainability of national immunization technical advisory groups (NITAGs) globally: lessons and recommendations from the founding meeting of the global NITAG network. Vaccine 2017;35(23):3007-11. DOI PubMed
2. Duclos P. National Immunization Technical Advisory Groups (NITAGs): guidance for their establishment and strengthening. Vaccine 2010;28 Suppl 1:A18-25. DOI PubMed

3. National Advisory Committee on Immunization (NACl). Evidence-based recommendations for immunization methods of the National Advisory Committee on Immunization. Can Commun Dis Rep 2009;35(ACS-1):1-10. https://www.canada.ca/content/dam/phac-aspc/migration/ phac-aspc/publicat/ccdr-rmtc/09pdf/ccdr-rmtc-vol-35-acsdcc-1.pdf

4. Influenza Working Group Members. An Advisory Committee Statement (ACS) National Advisory Committee on Immunization (NACl). Recommendations on the use of live, attenuated influenza vaccine $\left(\right.$ FluMist $\left.^{\circledR}\right)$ : supplemental statement on seasonal influenza vaccine for 2011-2012. Can Commun Dis Rep 2011;37(ACS-7):1-77. DOI

5. Grohskopf LA, Olsen SJ, Sokolow LZ, Bresee JS, Cox NJ, Broder KR, Karron RA, Walter EB; Centers for Disease Control and Prevention. Prevention and control of seasonal influenza with vaccines: recommendations of the Advisory Committee on Immunization Practices (ACIP) -- United States, 2014-15 influenza season. MMWR Morb Mortal Wkly Rep 2014;63(32):691-7. PubMed

6. National Advisory Committee on Immunization (NACl). Canadian Immunization Guide Chapter on Influenza and Statement on Seasonal Influenza Vaccine for 2016-2017: Addendum - LAIV use in children and adolescents. Ottawa (ON): PHAC; 2016 (updated 2018-02-21; accessed 2018-06-20). https://www.canada.ca/en/public-health/services/ immunization/national-advisory-committee-on-immunizationnaci/canadian-immunization-guide-chapter-on-influenzastatement-on-seasonal-influenza-vaccine-2016-2017addemdum-laiv-use-children-adolescents.html

7. National Advisory Committee on Immunization (NACl). An Advisory Committee Statement (ACS) - National Advisory Committee on Immunization (NACl): NACI opinion on LAIV effectiveness in young children. Ottawa (ON): PHAC; 2015 (updated 2015-02-24; accessed 2018-06-20). https://www. canada.ca/en/public-health/services/publications/healthyliving/naci-opinion-laiv-effectiveness-young-children.html

8. National Advisory Committee on Immunization (NACl). An Advisory Committee Statement (ACS) - National Advisory Committee on Immunization (NACl): Canadian Immunization Guide Chapter on Influenza and Statement on Seasonal Influenza Vaccine for 2015-2016. Ottawa (ON): PHAC; 2015 (updated 2016-03-08; accessed 2018-12-07). https://www. canada.ca/en/public-health/services/immunization/nationaladvisory-committee-on-immunization-naci/statement-onseasonal-influenza-vaccine-2015-2016.html

9. Grohskopf LA, Sokolow LZ, Olsen SJ, Bresee JS, Broder KR Karron RA. Prevention and Control of Influenza with Vaccines: Recommendations of the Advisory Committee on Immunization Practices, United States, 2015-16 Influenza Season. MMWR Morb Mortal Wkly Rep 2015;64(30):818-25. DOl PubMed 
10. Vaudry W, Stirling R; National Advisory Committee on Immunization (NACl). Summary of the NACI Statement on Seasonal Influenza Vaccine for 2017-2018. Can Commun Dis Rep 2017;43(5):96-103. DOI PubMed

11. Department of Health and Human Services Centers for Disease Control and Prevention CDC. Advisory Committee on Immunization Practices (ACIP): summary report. Atlanta (GA): US Centers for Disease Control and Prevention; 2016. https://www.cdc.gov/vaccines/acip/meetings/downloads/ min-archive/min-2016-06.pdf

12. Grohskopf LA, Sokolow LZ, Broder KR, Olsen SJ, Karron RA, Jernigan DB, Bresee JS. Prevention and Control of Seasonal Influenza with Vaccines Recommendations of the Advisory Committee on Immunization Practices-United States, 201617 influenza season. MMWR Recomm Rep 2016;65(5):1-54. DOI PubMed

13. Grohskopf LA, Sokolow LZ, Fry AM, Walter EB, Jernigan DB. Update: ACIP recommendations for the use of quadrivalent live attenuated influenza vaccine (LAIV4) - United States, 2018-19 Influenza Season. MMWR Morb Mortal Wkly Rep 2018;67(22):643-5. DOI PubMed

14. Kwong JC, Pereira JA, Quach S, Pellizzari R, Dusome E, Russell ML, Hamid JS, Feinberg Y, Winter AL, Gubbay JB, Sirtonski B, Moher D, Sider D, Finkelstein M, Loeb M; Public Health Agency of Canada/Canadian Institutes of Health Research Influenza Research Network (PCIRN) Program Delivery and Evaluation Group. Randomized evaluation of live attenuated vs. inactivated influenza vaccines in schools (RELATIVES) cluster randomized trial: pilot results from a household surveillance study to assess direct and indirect protection from influenza vaccination. Vaccine 2015;33(38):4910-5. DOI PubMed
15. Loeb M, Russell ML, Manning V, Fonseca K, Earn DJ, Horsman G, Chokani K, Vooght M, Babiuk L, Schwartz L, Neupane B, Singh P, Walter SD, Pullenayegum E. Live attenuated versus inactivated influenza vaccine in Hutterite children: a cluster randomized blinded trial. Ann Intern Med 2016;165(9):617-24. DOI PubMed

16. Tam TW. Intranasal influenza vaccine: why does Canada have different recommendations from the USA on its use? Paediatr Child Health 2018;23(1):31-4. DOI PubMed

17. Grohskopf LA, Alyanak E, Broder KR, Blanton LH, Fry AM, Jernigan DB, Atmar RL. Prevention and control of seasonal influenza with vaccines: recommendations of the Advisory Committee on Immunization Practices United States, 2020-21 Influenza Season. MMWR Recomm Rep 2020;69(8):1-24. DOI PubMed

18. National Advisory Committee on Immunization (NACl). An Advisory Committee Statement (ACS): Canadian Immunization Guide Chapter on Influenza and Statement on Seasonal Influenza Vaccine for 2021-2022. Ottawa (ON): PHAC; 2020 (modified 2021-01-28; accessed 2021-05-04). https://www.canada.ca/en/public-health/services/ publications/vaccines-immunization/canadian-immunizationguide-statement-seasonal-influenza-vaccine-2020-2021.html 